\title{
The role of faecal Candida albicans in the pathogenesis of food-intolerant irritable bowel syndrome
}

\author{
S.J. Middleton, A. Coley and J.O. Hunter \\ Department of Gastroenterology, Addenbrooke's Hospital, Cambridge CB2 2QQ, UK
}

\begin{abstract}
Summary: Candida albicans was sought in stool samples from 38 patients with irritable bowel syndrome and 20 healthy controls. In only three patients with irritable bowel syndrome was $C$. albicans discovered and these patients had either recently received antibiotics or the stool sample had been delayed more than 24 hours in transit. $C$. albicans was isolated from none of the control stool samples. We conclude that $C$. albicans is not involved in the aetiology of the irritable bowel syndrome.
\end{abstract}

\section{Introduction}

The irritable bowel syndrome (IBS) is the most common gastroenterological disorder in clinical practice in Western society. ${ }^{1,2}$ It is now known that many patients with IBS have specific food intolerances. ${ }^{3,4}$ Diets have enabled many patients to control their symptoms but limited diets are expensive and socially inconvenient. C. albicans has been implicated in antibiotic-associated diarrhoea in elderly patients $s^{5}$ and it has been suggested that food intolerance may be a consequence of the overgrowth of $C$. albicans in the gut. ${ }^{6}$ This theory has received much publicity in the popular press but little scientific analysis. In this study we report the results of a search for $C$. albicans in the stools of patients with IBS proven to be caused by food intolerance and in those of normal healthy controls.

\section{Materials and methods}

\section{Patients}

The diagnosis of IBS was based upon the standard Manning criteria following exclusion of organic disease by a combination of investigations including normal stool culture, full blood count, ESR, acute phase proteins, plasma, glucose, liver function tests, urea, creatinine, electrolytes and amylase, together, where clinically indicated, with lactose tolerance test, barium enema, abdominal ultrasound, intestinal permeability and radiolabelled granulocyte scans. Food intolerance was

Correspondence: J.O. Hunter, F.R.C.P.

Accepted: 17 January 1992 detected by the relief of symptoms following two weeks on a standard exclusion diet. ${ }^{7}$ Patients subsequently reintroduced foods to see which if any precipitated their symptoms and the intolerances thus discovered were confirmed by double blind challenge. Normal volunteers were recruited from hospital staff and patients relatives who were in the same age range as the patients studied.

\section{Microbiological investigations}

Stool samples were collected by patients using a wooden spatula and $20 \mathrm{ml}$ metal container with screw top and delivered to the laboratory within 24 hours. Validation of $C$. albicans viability during transport was performed by seeding $1 \mathrm{~g}$ samples of C. albicans-negative stool with a known concentration of the organism and incubating these for 24 hours at various temperatures. The number of colony-forming units present on Sabouraud's dextrose agar was determined for each sample. A 10-fold increase in C. albicans counts occurred at $22^{\circ} \mathrm{C}$ and $37^{\circ} \mathrm{C}$, but not at $4^{\circ} \mathrm{C}$, which remain unchanged. On the receipt of faecal samples, $1 \mathrm{~g}$ of homogenized faeces was cultured on Sabouraud's medium. A colony count was performed after $\mathbf{4 8}$ hours of aerobic incubation. Colonies of Candidalike organisms were incubated in horse serum for 3 hours at $37^{\circ} \mathrm{C}$, and a wet film was then examined for filamentous overgrowth or 'germ tubes', a finding specific for C. albicans. ${ }^{8}$

\section{Results}

There were no $C$. albicans colonies detected after incubating the faeces of the control group. Moderate numbers of $C$. albicans (approximately $10^{4} / \mathrm{g}$ 
wet faeces) were grown from faecal samples taken from three IBS patients. Two of the three had received antibiotic therapy near the time of sampling and the third faecal specimen had been exposed to an extended period of transit following collection prior to incubation. On resampling these individuals there were no $C$. albicans colonies seen after 48 hours incubation.

\section{Discussion}

IBS is not a homogeneous entity but a collection of poorly understood conditions presenting with similar symptoms, namely abdominal pain and an abnormal bowel habit. It is now clear that in the United Kingdom approximately half the patients diagnosed as IBS may be benefitted by dietary manipulation, at least temporarily. The pathogenesis of such food intolerance is still unknown. ${ }^{9}$ However, it has been suggested that changes in the bacterial flora of the gut may be a factor involved. Other workers have suggested that overgrowth of C. albicans in the colonic flora is a common feature of patients suffering food intolerance. ${ }^{6,10}$ They have advocated treatment with so-called 'yeast-free diets' and antifungal drugs, such as nystatin and ketoconazole. As these drugs have caused harmful

\section{References}

1. Ferguson, A., Sircus, W. \& Eastwood, M. Frequency of 'functional' gastrointestinal disorders. Lancet 1977, ii: 613.

2. Painter, N.S. The high fibre diet in the treatment of diverticular disease of the colon. Postgrad Med J 1974, 50: 629-635.

3. Alun Jones, V., McLaughlan, P., Shorthouse, M., Workman, E. \& Hunter, J.O. Food intolerance: a major factor in the pathogenesis of irritable bowel syndrome. Lancet 1982, ii: 1115-1117.

4. Nanda, R., James, R., Smith, H., Dudley, C.R.K. \& Jewell, D.P. Food intolerance and the irritable bowel syndrome. Gut 1989, 30: 1099-1104.

5. Danna, P.L., Urban, C., Bellin, E. \& Rahal, J.J. Role of candida in pathogenesis of antibiotic-associated diarrhoea in elderly patients. Lancet 1991, i: 511-514.

6. Crook, W.G. The Yeast Connection, 2nd ed. Professional Books, Jackson, Tennessee, 1984. side effects, ${ }^{11,12}$ it is important to establish whether or not C. albicans in the gut is a significant factor producing symptoms in these patients.

We were concerned that the delay in culturing the stools of our patients caused by transportation to the laboratory might lead to reduced $C$. albicans counts. However, the studies we have performed in which stools were spiked with the organism and then cultured after incubation for 24 hours after collection at $4^{\circ} \mathrm{C}, 22^{\circ} \mathrm{C}$ and $37^{\circ} \mathrm{C}$ showed that this did not occur.

We found $C$. albicans to be no more prolific in the faeces of people with IBS than those of our healthy controls. Our results are similar to those found on review of 168 patients who claimed that their symptoms were related to Candida in the gut. As in our study, no evidence was found to substantiate an association between symptoms and $C$. albicans infection. ${ }^{13}$ Anecdotal reports of improvement after treatment for $C$. albicans may be a consequence of simultaneous dietary measures (the 'yeast-free diet') in patients who are food intolerant. ${ }^{3,4}$

Overgrowth of $C$. albicans is not the cause of food intolerance in patients with IBS and anticandidal drugs should not be used in the treatment of this condition.

7. Workman, E., Alun Jones, V. \& Hunter, J.O. The Allergy Diet. Dunitz, London, 1984.

8. Douglas Sleigh, J. \& Timbury, M.C. Candida. In: Notes on Medical Bacteriology. Churchill Livingstone Medical Text, Edinburgh, 1981, p. 133.

9. Hunter, J.O. Food allergy - or enterometabolic disorder? Lancet 1991, ii: $495-496$

10. Galland, L. Nutrition and candidiasis. J Orthomol Psychiat 1985, 14: 50-60.

11. Lewis, J.H., Zimmerman, H.J., Benson, G.D. et al. Hepatic injury associated with ketoconazole therapy. Gastroenterology 1984, 86: 503.

12. Quinn, J.P. \& Venezio, F.R. Ketonozole and the yeast connection. JAMA 1986, 255: 3250.

13. Renford, L. et al. Yeast connection among 100 patients with chronic fatigue. Am J Med 1989, 86: 165-168. 